\title{
Recurrent meningitis by Streptococcus pneumoniae in a girl with cochlear implant and head trauma despite 13-valent conjugated pneumococcal vaccine
}

\author{
Melike Emiroglu M. D. ${ }^{a}$, Gulsum Alkan M.D. ${ }^{a}$, Bahadır Feyzioglu M.D. ${ }^{b}$, Ahmet E. Aycan Biol. ${ }^{c}$ and \\ Mehmet Ceyhan M.D. ${ }^{\circ}$
}

\begin{abstract}
Recurrent bacterial meningitis is a very rare phenomenon in children. Skull base fractures and cochlear implant are the important predisposing factors and, Streptococcus pneumoniae is the most frequently isolated agent. Implementation of 13-valent conjugated pneumococcal vaccine (PCV13) has reduced the occurence of invasive pneumococcal diseases. Vaccination breakthrough is typically related to underlying predisposing conditions. Herein, we reported recurrent pneumococcal meningitis in a patient with a cochlear implant whoexperienced a head trauma after being fully vaccinated with PCV13. The patient experienced three meningitis episodes within one year. S.pneumoniae was determined on CSF culture in the first and third episodes and detected by PCR at the second episode. Neurosurgical intervention was performed after the third meningitis episode, and the patient had no recurrence problems for the following two years. To our knowledge, breakthrough S.pneumoniae serotype 1 meningitis after full PCV13 immunization has not been reported elsewhere in the literature.
\end{abstract}

Key words: Streptococcus pneumoniae, recurrent meningitis, craniocerebral trauma, vaccination, child.

http: / / dx.doi.org/10.5546/ aap.2019.eng.e373

To cite: Emiroglu M, Alkan G, Feyzioglu B, Aycan AE, Ceyhan M. Recurrent meningitis by Streptococcus pneumoniae in a girl with cochlear implant and head trauma despite 13-valent conjugated pneumococcal vaccine. Arch Argent Pediatr 2019;117(4):e373-e376. a Selcuk University Faculty of Medicine, Department of Pediatric Infectious Diseases, Konya, Turkey.

b. Necmettin Erbakan University Faculty of Medicine, Department of Medical Microbiology, Konya, Turkey.

c. Hacettepe University Pediatric Infectious Diseases Department, Ankara, Turkey.

E-mail address:

Gulsum Alkan, M.D.: galkan-85@hotmail.com

Funding: None.

Conflict of interest: None.

Received: 3-9-2018

Accepted: 1-21-2019

\section{INTRODUCTION}

Streptococcus pneumoniae (S. pneumoniae) is characterized over 91 serotypes according to its polysaccharide capsules. Approximately 13 serotypes are responsible for more than $75 \%$ of invasive pneumococcal diseases (IPD), such as meningitis, sepsis, pneumonia, and bacteremia in children. ${ }^{1}$ Different serotypes exhibit different clinical expression of the disease. It is unknown whether disease severity is the result of capsule type or other virulence factors. ${ }^{2}$ S. pneumoniae is the most common bacterial meningitis agent in cases with cerebrospinal fluid (CSF) leakage and cochlear implants. ${ }^{3}$ Conjugated pneumococcal vaccines are very effective at preventing severe diseases, including meningitis, hospitalization and even death. PCV7, which includes the $4,6 \mathrm{~B}, 9 \mathrm{~V}, 14,18 \mathrm{C}$, $19 \mathrm{~F}$ and $23 \mathrm{~F}$ serotypes, was introduced into the Turkish national immunization program in 2009. In 2011 was replaced with PCV13, which also includes the 1,3, 5, 6A, 7F and 19A serotypes. In a multicenter study from Turkey (2010), the PCV7, PCV10, and PCV13 coverage rates for bacterial meningitis were $48.1 \%, 85.2 \%$, and $92.3 \%$, respectively, in all age groups. ${ }^{4}$ Despite the effectiveness of vaccine, vaccination failure (VF) has been observed in children with underlying predisposing conditions. We reported recurrent pneumococcal meningitis caused by serotype 1 in a fully vaccinated child with cochlear implants after a head trauma.

\section{CASE REPORT}

A 4,5 year-old girl presented with fever, headache and somnolance. When she was 18 months of age, she underwent right cochlear implantation with positioner for inner ear malformations. She suffered skull trauma without any otorrhea or rhinorrhoea. Image of brain tomography showed a linear fracture in right occipital bone. Sixth months after the trauma, she experienced her first meningitis episode (age of 4 years) due to S. pneumoniae penicillinsusceptible, which was detected by CSF culture. 
Although she had been vaccinated with PCV13 at $2,4,6$, and 12 months of age and, she received one dose of PPV23 after first pneumococcal meningitis episode.

At admission physical examination, meningeal irritation signs and papilledema were positive, while otherwise normal. Lumbal puncture was performed on day two. Cerebrospinal fluid (CSF) analysis revealed 100 cells $/ \mathrm{mm}^{3}$ with neutrophilia; protein and glucose contents were $21 \mathrm{mg} / \mathrm{dL}$ and $53 \mathrm{mg} / \mathrm{dL}$, respectively. Her primary laboratory data were: white blood cell counts $32,600 / \mathrm{mm}^{3}$, neutrophils $93 \%$; hemoglobin $11 \mathrm{~g} / \mathrm{dL}$; platelets $411,000 / \mathrm{mm}^{3}$; erythrocyte sedimentation rate (ESR), $36 \mathrm{~mm} / \mathrm{h}$ and C-reactive protein (CRP) $95 \mathrm{mg} / \mathrm{L}$. While CSF culture was negative, CSF PCR was positive for S. pneumoniae. The patient was treated with ceftriaxon and vancomycin for 14 days. Her postreatment magnetic resonance cisternography was normal.

Six months later (age of 5 years), the patient was admitted again with fever, headache, and vomiting. Her physical examination was normal, except neck stiffness and irritiability. The CSF cell count was 1000 neutrophils $/ \mathrm{mm}^{3}$, and the protein and glucose contents were $219 \mathrm{mg} / \mathrm{dL}$ and $12 \mathrm{mg} / \mathrm{dL}$, respectively. Blood examination showed: WBC 32,000/ $\mathrm{mm}^{3}$ with $85 \%$ neutrophils,

FIGURE 1. Cerebrospinal fluid leakage from the right petrooccipital region into the middle ear

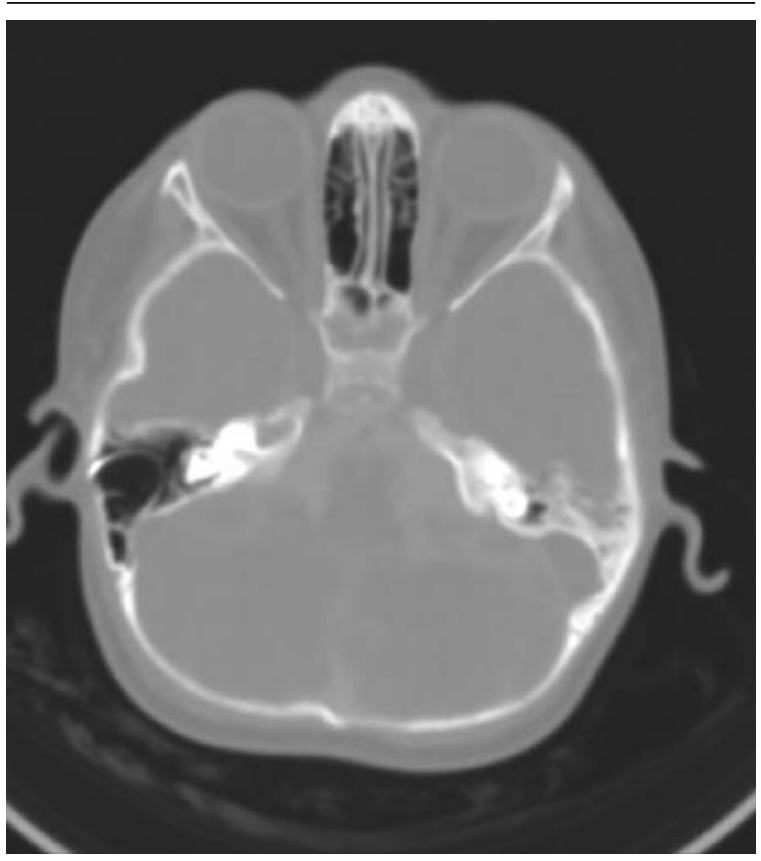

ESR $36 \mathrm{~mm} / \mathrm{hr}$ and CRP $94 \mathrm{mg} / \mathrm{L}$. Innitially vancomycin and ceftriaxon were given. Her CSF culture showed growth of penicillin-susceptible S. pneumoniae. Ceftriaxone treatment was administered for 14 days.

The 3 episodes of meningitis were treated successfully without complications. Control CSF examinations were normal after two weeks of treatment. Isolates from the patient's first and third meningitis episodes were identified as serotype 1 based on the Quellung reaction using serotype-specific antisera (Statens Serum Institute, Copenhagen, Denmark).

Immunologic screening tests (blood cell counts, peripheral blood lymphocyte subsets, serum total immunoglobulin levels, dihydrorhodamine test, complement system) were all in normal limits; ELISA for human immunodeficiency virus was negative. Total anti-pneumococcal IgG was detected as $2.8 \mathrm{microgr} / \mathrm{mL}$.

Abdomen ultrasound confirmed the presence of a spleen. Computed tomography (CT) cysternography was performed again, and CSF leakage originating from the right petro-occipital region into the middle ear was confirmed (Figure 1). After antibiotic treatment was completed, surgical repairment was done. The patient had no recurrences for the following two years.

\section{DİSCUSSİON}

Recurrent bacterial meningitis (RBM) is defined as two or more episodes of pyogenic meningitis separated by a period of convalescence and the complete resolution of all signs. Although the fistulous connection between nasopharyngeal flora and the CSF space after head trauma poses a significant risk for recurrent meningitis, anatomical defects, especially in the inner ear, cochlear implant and immunodeficiency disorders are other prominent predisposing factors. ${ }^{5}$ S. pneumoniae is the most common causative agent in recurrent bacterial meningitis after head trauma, followed by $H$. influenzae and N. meningitidis. ${ }^{6}$

Different serotypes of S. pneumoniae exhibit different clinical expression of the disease. Specifically, serotype 1 is considered highly invasive and causes more bacteremic pneumonia than meningitis. ${ }^{7}$ Clear relationships between some pneumococcus serotypes and organ involvements exist, such as the relationship between serotype 1, 3, 14, 15, 19A, 33 and necrotizing pneumonia and empyema. However, no study has found a relationship between a 
special serotype and meningitis due to CSF leakeage or an inner ear anomaly. ${ }^{8}$

PCV are highly effective in preventing IPD due to serotypes included in the vaccines. VF is rare, irrespective of vaccine or schedule. Von Mollendorf et al. reported that the serotype 1 disease nearly disappeared among children after PCV13 introduction. ${ }^{7}$

Vaccination breakthrough (VBT) is defined as a pneumococcal meningitis with PCV serotype in a child who had received one or more than PCV doses, and VF is defined as a subset of VBT that occurs in a child who completed the PCV series. ${ }^{9}$ Godot et al. conducted a study from 2003 to 2013 about recurrent pneumococcal meningitis after the implementation of PCVs, in France. VBT accounted for $3.2 \%$ of the cases (PCV7 era: 24 of 943 , PCV13 era: 15 of 290) and VF $0.6 \%$ (PCV7 era: 6 of 943, PCV13 era: 2 of 290). ${ }^{9}$ VBT may be the result of the infection patogenesis or the immune system's responsiveness to the vaccine. Conjugated vaccines are very effective for serious diseases, such as bacteraemia and local infection. Rats could not be fully protected with vaccination from meningitis when the bacteria were inoculated directly into the inner ear. ${ }^{10,11}$

Significant variation occurs in the serotypespecific immune response to PCV. In Barrow et al. study, in 107 children who had received PCV7, serotype-specific antibodies were measured. Eight children failed to develop a response to their infecting serotype $[4,6 \mathrm{~B}(\mathrm{n}=4), 14,18 \mathrm{C}$ $(n=2]$ despite receiving at least 3 doses vaccine. ${ }^{12}$ This phenomenon may be the result of large pneumococcal polysaccharide loads that deplete the memory B-cell pool and cause immune paralysis, or potential genetic basis for nonresponse to individual pneumococcal serotypes. ${ }^{13}$

Additionally, in patients with RBM, comorbidity is considered to be high among VF cases, but there is limited data. Godot et al. reported that only 10 of 47 patients with RBM and VBT had meningeal breach ( 2 congenital and 2 posttraumatic), cochlear implant $(n=1)$, immunodeficiencies $(n=5)$. In these 10 patients, serotype $19 \mathrm{~F}(\mathrm{n}=6)$ was the most frequent cause, even after the introduction of PCV13. Other serotypes identified in the study were $6 \mathrm{~B}(\mathrm{n}=2)$, $4(\mathrm{n}=1), 23 \mathrm{~F}(\mathrm{n}=1) .{ }^{9} \mathrm{Li}$ et al., reported recurrent pneumococcal meningitis serotype $23 \mathrm{~F}$ in a child with CSF leakage who was fully vaccinated with PCV13. ${ }^{14}$

Different techniques can use for detect of locating CSF fistulas. MR cisternography showed sensitivity of $92 \%$ and specificity of $100 \%$. The primary disadvantages of MRI are its poor spatial resolution compared with $\mathrm{CT}$ and the absence of bony details.

MRI accurately detects CSF leaks and associated complications such as encephaloceles and meningoceles. This may be the cause of diagnosis delay in our patient. ${ }^{15}$

We assumed our patient as VF, because she experienced recurrent meningitis due to serotype 1 despite being fully vaccinated with PCV13 and PPV which covered serotype 1 . The infection depend on spreading from middle ear to her subarachnoid area. Unfortunately, we could not determine the serotype-specific antibody levels or obtain a culture from the middle ear.

When evaluating a child with recurrent meningitis, predisposing factors must be tested for CSF leakage, immune deficiency or indwelling devices, regardless of the child's immunization status.

\section{REFERENCES}

1. Cohen R, Biscrdi S, Levy C. The multifaceted impact of pneumococcal conjugate vaccine implementation in children in France between 2001 to 2014. Hum Vaccin Immunother. 2016; 12(2):277-84.

2. Lynch JP 3rd, Zhanel GG. Streptococcus pneumoniae: Epidemiology, Risk Factors, and Strategies for Prevention. Semin Respir Crit Care Med. 2009; 30(2):189-209.

3. Santos SF, Rodrigues F, Dias A, Costa JA, et al. Meningite bacteriana pós-traumática em idade pediátrica: análise de onze anos. Acta Med Port. 2011; 24(3):391-8.

4. Ceyhan M, Yildirim I, Sheppard CL, George RC. Pneumococcal serotypes causing pediatric meningitis in Turkey: application of a new technology in the investigation of cases negative by conventional culture. Eur J Clin Microbiol Infect Dis. 2010; 29(3):289-93.

5. Ghosh A, Sharma S, Halder PP, Bhattacharya A, Mukherjee S. Diagnostic Dilemma in a 15-Year-Old Boy with Recurrent Pneumococcal Meningitis. J Clin Diagn Res. 2017; 11(5):SD03-4.

6. Tebruegge M, Curtis N. Epidemiology, etiology, pathogenesis, and diagnosis of recurrent bacterial meningitis. Clin Microbiol Rev. 2008; 21(3):519-37.

7. von Mollendorf C, Cohen C, Tempia S, Meiring S, et al. Epidemiology of serotype 1 invasive pneumococcal disease, South Africa, 2003-2013. Emerg Infect Dis. 2016; 22(2):26170.

8. Bender JM, Ampofo K, Korgenski K, Daly J, et al. Pneumococcal necrotizing pneumonia in utah: does serotype matter? Clin Infect Dis. 2008; 46(9):1346-52.

9. Godot C, Levy C, Varon E, Picard C, et al. Pneumococcal meningitis vaccine breakthroughs and failures after routine 7-valent and 13-valent pneumococcal conjugate vaccination in children in France. Pediatr Infect Dis J. 2015; 34(10):e260-3.

10. O'Mahony LN, Klein EJ, Walker W. A fully immunized child with a cochlear implant and streptococcus pneumoniae meningitis 3 years after implantation. Pediatr Emerg Care. 2011; 27:(3):200-2. 
11. Sjöström K, Spindler C, Ortqvist A, Kalin M, et al. Clonal and capsular types decide whether pneumococci will act as a primary or opportunistic pathogen. Clin Infect Dis. 2006; 42(4):451-9.

12. Borrow R, Stanford E, Waight P, Helbert M, et al. Serotypespecific immune unresponsiveness to pneumococcal conjugate vaccine following invasive pneumococcal disease. Infect Immun. 2008; 76(11):5305-9.
13. Stanford E, Ladhani S, Slack M, Scott D, et al. Pneumococcal serotype-specific unresponsiveness in vaccinated child with cochlear implant. Emerg Infect Dis. 2012; 18(6):1024-6.

14. LiYC, Chen CY, Wu KH, KuoHT, Wu HP. Recurrent Streptococcus Pneumoniae $23 \mathrm{~F}$ meningitis due to cerebrospinal fluid leakage from the ear cannel: a case report. $B M C$ Pediatr. 2015; 15:195.

15. Vemuri NV, Karanam LSP, Manchikanti V, Dandamudi $\mathrm{S}$, et al. Imaging review of cerebrospinal fluid leaks. Indian J Radiol Imaging. 2017; 27(4):441-6. 